\title{
Immunohistochemical analysis of melanocyte content in different zones of vitiligo lesions using the Melan-A marker
}

\author{
Alexey Kubanov ${ }^{1,2}$, Diana Proshutinskaia ${ }^{1,2 \bowtie}$, Vladimir Volnukhin ${ }^{1}$, Oksana Katunina ${ }^{1}$, Tatiana Abramova ${ }^{2}$
}

\begin{abstract}
Introduction: Occurence of vitiligo lesions is caused by the destruction of melanocytes in affected skin and therefore by the reduction of pigment melanin content. Questions remain about the presence of residual melanocytes in the depigmented skin and optimal methods of their identification.

Methods: Skin biopsy samples from 16 patients with non-segmental vitiligo and from 10 healthy volunteers were investigated for Melan-A (A103 clone)+ melanocytes expression by immunohistochemical analysis and for melanin by histochemical studies with section staining by Fontana-Masson method.

Results: For some patients including those with long-standing disease (up to 40 years) Melan-A+ cells and melanin granules were detected in depigmented skin as indication that the residual melanocytes are preserved in vitiligo lesions. More than three-fold decrease of Melan-A+ melanocytes amount was revealed in perilesional normally pigmented skin of vitiligo patients ( $<<0.001)$ compared with the skin of healthy volunteers. Clinically intact skin involvement in the pathological process should be taken into consideration if local treatment methods are prescribed.

Conclusion: In some vitiligo patients the residual melanocytes are preserved in depigmented skin. Melan-A marker is useful for identification of melanocytes in vitiligo patients' skin.
\end{abstract}

Keywords: immunohistochemistry, melanocytes, Melan-A marker, vitiligo

Received: 20 November 2015 | Returned for modification: 24 December 2015 | Accepted: 18 January 2016

\section{Introduction}

Vitiligo is a chronic disease of unknown etiology characterized by the onset of depigmented spots on various body areas. Currently, the generally accepted hypothesis of the cause of vitiligo is the autoimmune one, according to which the destruction of melanocytes in vitiligo lesions is induced by autoimmune mechanisms (1-3).

In 1991, Cui et al. showed that the depigmented skin of vitiligo patients carries residual melanocytes that can be preserved in hair follicles for a long time (4). These melanocytes are able to act as a reservoir for the repopulation of pigment cells with normal function during disease treatment.

According to some authors, epidermal melanocytes are absent in depigmented skin (5-7). According to others, melanocytes and melanin are found in the area of depigmentation even in longstanding vitiligo lesions (8-10). Such ambiguous results may to some extent be due to differences in markers and research methods.

Immunohistochemical research on various melanocytic antigen expression in the skin provides important information about melanocyte status in vitiligo lesions. Several different markers have been suggested for melanocyte identification, but none of them show absolute specificity and sensitivity (11).

One of the most important melanocytic markers is the MelanA marker, also known as MART-1 (melanoma antigen recognized by $\mathrm{T}$ cells-1). This marker is expressed in melanosomes and the endoplasmic reticulum of melanocytes, and it identifies the melanosomal protein that participates in formation and maturation of melanosomes (12). Forming a complex with Pmel17 protein (also known as gp1oo or "silver locus" product), Melan-A plays an essential role in its expression, stability, transport, and processing in melanosomes (13). In turn, Pmel17 protein (identified by HMB45, HMB-50, and NKI-beteb markers) acts as the main structural protein of the internal matrix fibers of melanosomes.

Melan-A antigen is mostly expressed in melanocytes of the skin and the retina (14). Studying Melan-A expression in vitiligo lesions is of significant interest because this melanosomal protein is one of the immunogenic determinants of melanocytes to which autoreactive circulating CD8+ T-cells were found in patients' blood (15-17). Melan-A-specific cytotoxic T-cells are known to have the phenotype of memory T-cells (CD45RO+) and to express cutaneous lymphocyte antigen (CLA) (15), and so their important role in vitiligo pathogenesis is evident.

The purpose of this study was to investigate the usefulness of the Melan-A marker for identifying and estimating melanocyte content in different zones of non-segmental vitiligo lesions: the zone of depigmented skin, marginal zone (bordering the area), and zone of perilesional normally pigmented skin.

\section{Material and methods}

Immunohistochemical and histochemical analyses were performed for 16 patients with non-segmental vitiligo and for 10 healthy volunteers. In vitiligo patients, skin biopsy material was taken from three zones: the zone of depigmented skin, marginal zone (bordering the area), and zone of perilesional normally pigmented skin.

In healthy volunteers, skin samples taken during cosmetic procedures were used as study material. Skin biopsies were fixated in a buffered $10 \%$ formalin solution, processed, and embedded in paraffin. Paraffin blocks were cut into 4 - to 5-micron sections on a microtome and placed on glass slides.

Immunohistochemical studies were performed with pre-treat- 
ment demasking in a microwave oven and using a Novostain Universal Detection Kit detection system (Novocastra Laboratories Ltd., UK). Mouse monoclonal Melan-A antibodies, A103 clone (Novocastra Laboratories Ltd., UK) were used for the phenotyping at a dilution of 1:25. Deparaffinization of the sections in xylene was followed by the demasking of antigens by boiling in a citrate buffer (pH 6.o) using a microwave oven for three cycles, 5 minutes each, with a 1-minute break between each cycle. After cooling, the slides were washed in two changes of TRIS-buffer ( $\mathrm{pH} 5.54$ ). In order to prevent endogenic peroxidase activity, a solution of $0.3 \%$ hydrogen peroxide with methanol (1:1 ratio) was applied to the sections. Hematoxylin staining of nuclei was performed in all cases.

Melanin granules in the skin were detected through FontanaMasson histochemical staining of the sections.

The prepared specimens were studied using a Nikon Eclipse E 600 light microscope and photographed with a Nikon D1oo digital camera. The amount of Melan-A+ cells was detected in the basal layer of the epidermis, and the amount of melanin-containing cells was detected in the basal and suprabasal layers, the calculation being made per 100 basal keratinocytes. Immunoreactive cells were counted in five fields of vision, and after that the average value was calculated for each specimen.

Statistical analysis was performed using the software package Statistica 6.1 (StatSoft, Inc., USA). Descriptive statistics of quantitative traits was presented as medians and quartiles (Me [Q1; Q3]). The Mann-Whitney U-test was used for comparison of unrelated groups by both quantitative and ordinal traits. Correlation analysis was performed using the Spearman rank correlation coefficient. In testing hypotheses, the differences were considered statistically significant at $P<0.05$.

\section{Results}

The 16 patients examined with non-segmental vitiligo included 11 women and five men. The patients were between 24 and 52 years old (median 38 years), the duration of the disease was between 21 months and 42 years (median 16 years), and the affected area was between 5 and 50\% of the body surface (median 20\%). All patients had a progressive (unstable) disease stage. The control group consisted of 10 healthy volunteers between 29 and 64 years old (median value 46 years). The group of patients and group of healthy volunteers were not statistically different in age and sex ( $P=0.200$ and $P=0.130$, respectively).

In the group of healthy volunteers, Melan-A+ melanocytes and melanin granules were found in all skin samples examined. Melan-A+ cells were revealed in the basal layer of the epidermis (Fig. 1), and melanin-containing cells were presented in the cells of the basal and suprabasal layers of the epidermis (Fig. 2).

In some vitiligo patients, expression of Melan-A melanosomal protein and melanin granules was detected in all three zones of vitiligo lesions. In the zone of depigmented skin, Melan-A+ melanocytes and melanin granules were found in six and nine patients, respectively; in the marginal zone, in 13 and 15 patients, respectively; and in the zone of perilesional normally pigmented skin, in all patients examined. For patients with detected Melan$\mathrm{A}+$ and melanin-containing cells in the zone of depigmented skin, duration of the disease ranged from 21 months to 40 years.

In the patients' skin, Melan-A expression was evident in the cells of basal layer of the epidermis: in the zone of depigmented

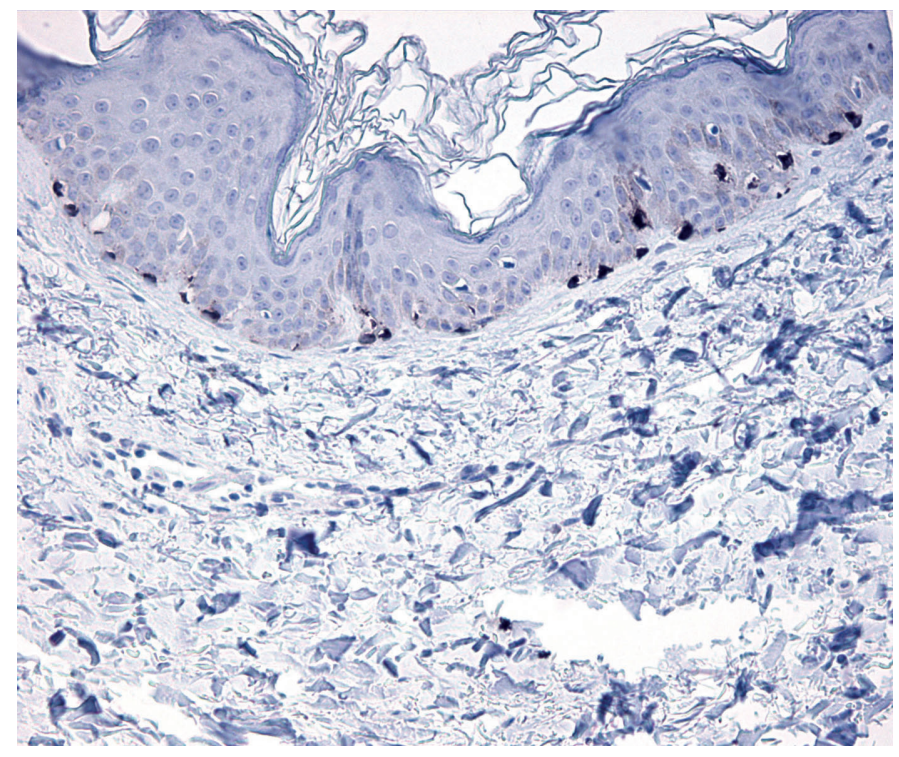

Figure 1 | Expression of Melan-A in the skin of a healthy volunteer. Immunohistochemical reaction with monoclonal antibodies A103, $\times 200$.

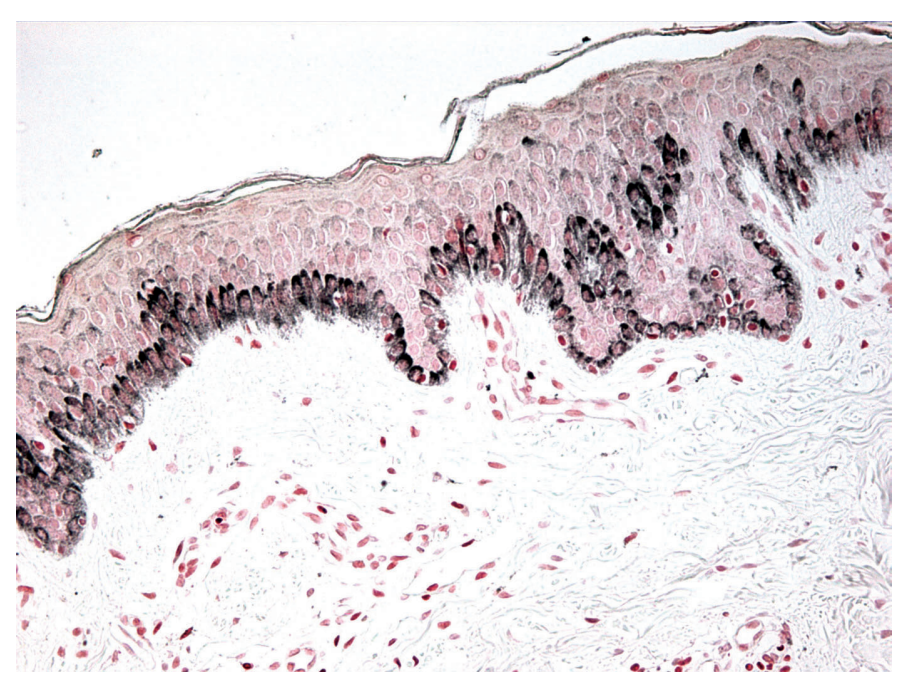

Figure 2 | Melanin granules in the skin of a healthy volunteer. Fontana-Masson staining, $\times 200$.

skin, individual stained cells were detected (Fig. 3a), whereas in the marginal zone and in the zone of perilesional normally pigmented skin the amount varied from one to seven cells per 100 keratinocytes of the basal layer (Figs. 3b and 3c). Regarding melanin granules, in the zone of depigmented skin they were found in individual regions of the basal layer, in the marginal zone they were found in almost the entire basal layer, and in the zone of perilesional normally pigmented skin they were found throughout the basal and suprabasal layers of the epidermis (Figs. 4a-c).

Statistical analysis showed a decreased amount of Melan-A+ cells in all three areas of vitiligo lesions compared with the skin of healthy volunteers $(P<0.001)$ (Table 1$)$; moreover, its amount in the zone of perilesional normally pigmented skin was over three times lower.

The amount of melanin-containing cells significantly decreased in the zone of depigmented skin $(P<0.001)$ and in the marginal zone $(P=0.013)$, whereas in the zone of perilesional normally pigmented skin it did not differ from the value in the skin of healthy volunteers. In the zone of depigmented skin and in the marginal zone, the amount of Melan-A+ melanocytes correlated with the amount of melanin-containing cells: $r=0.75(P<0.001)$ and $\mathrm{r}=0.79(P<0.001)$, respectively. 

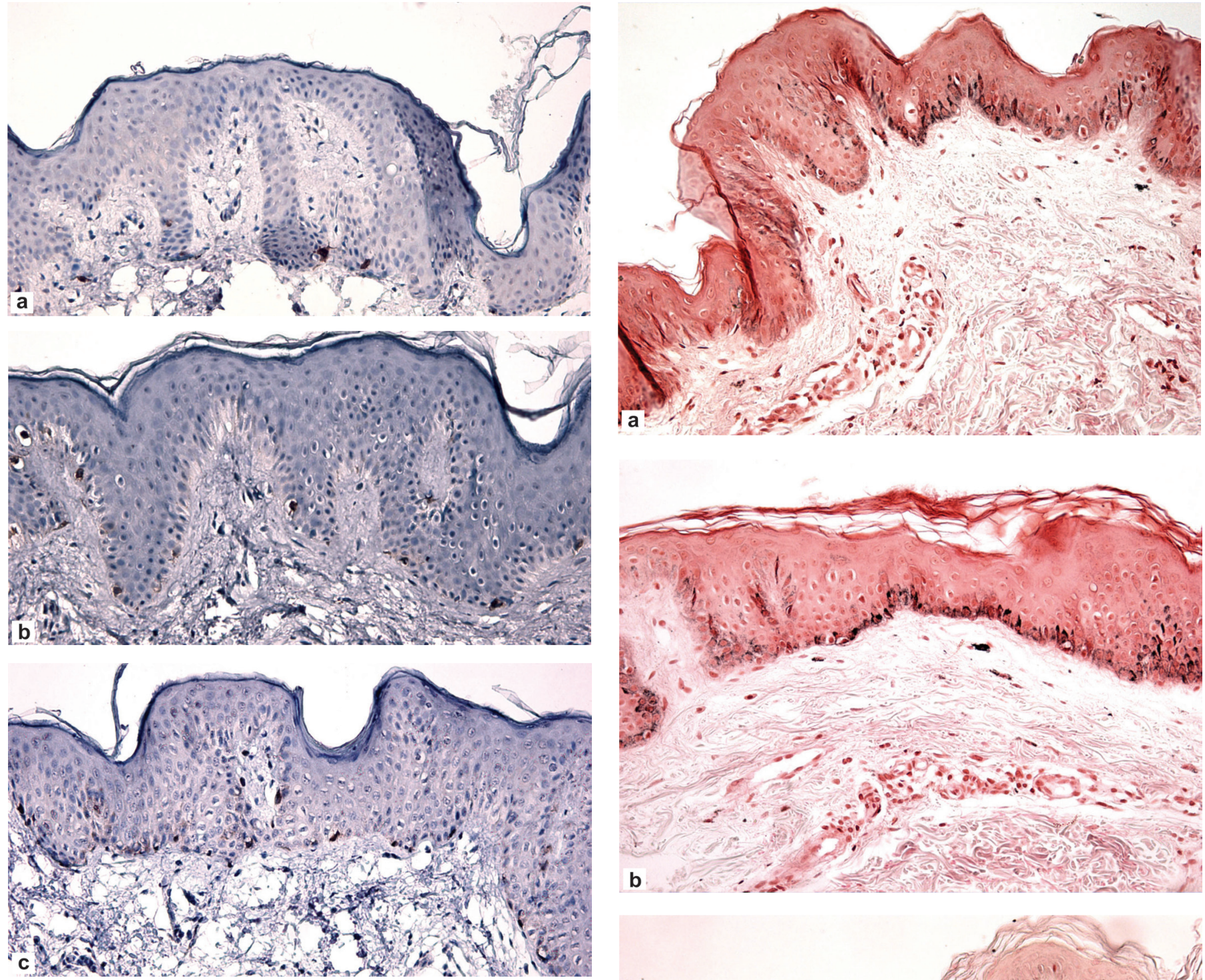

Figure 3 | a: Expression of Melan-A in the skin of a vitiligo patient. Immunohistochemical reaction with monoclonal antibodies A103, $\times 200$. Zone of depigmented skin. $\mathbf{b}$. Expression of Melan-A in the skin of a vitiligo patient. Immunohistochemical reaction with monoclonal antibodies A103, $\times 200$. Marginal zone. c. Expression of Melan-A in the skin of a vitiligo patient. Immunohistochemical reaction with monoclonal antibodies $\mathrm{A} 103, \times 200$. Zone of perilesional normally pigmented skin.

\section{Discussion}

In recent years, the Melan-A marker has been increasingly used in diagnosing various pigment neoplasms and diseases of the skin (18). It is well expressed in melanocytes of healthy skin (12, 19, 20) and transformed melanocytes of benign and malignant neoplasms of melanocytic genesis (21-25).

In a number of studies, the Melan-A marker was shown to be more specific and sensitive than the S-100 and HMB-45 melanocytic markers (14, 20, 22-24). The S-10o marker, which identifies the protein S-10o, is not specific enough because it is expressed not only

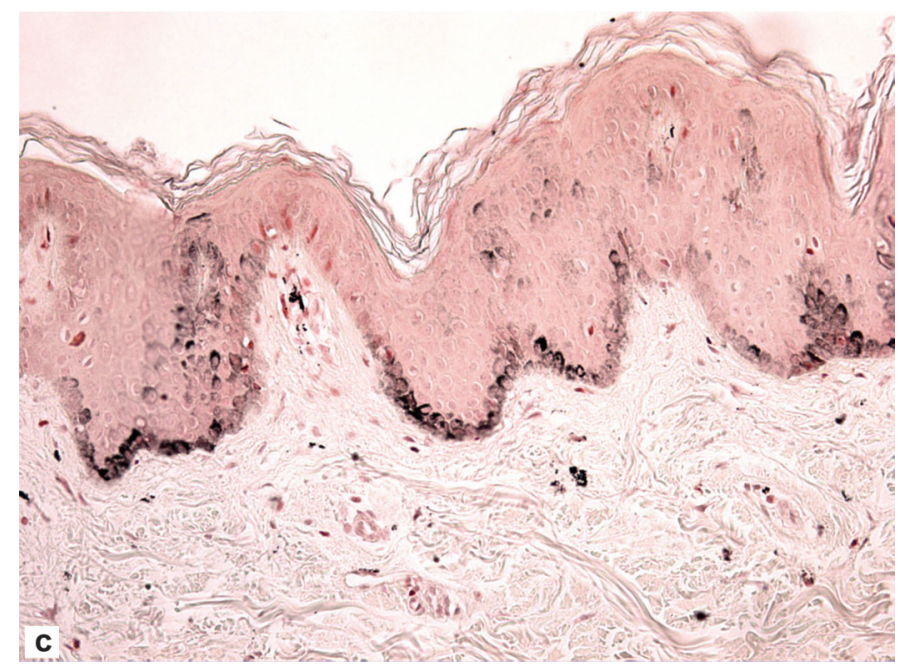

Figure 4 | a: Melanin granules in the skin of a vitiligo patient. Zone of depig mented skin. Fontana-Masson staining, $\times 200$. b. Melanin granules in the skin of a vitiligo patient. Marginal zone. Fontana-Masson staining, $\times 200$. c. Melanin granules in the skin of a vitiligo patient. Zone of perilesional normally pigmented skin. Fontana-Masson staining, ×200.

Table 1 | Content of Melan-A+ melanocytes and FM+ cells in the epidermis of patients with vitiligo and healthy volunteers (amount of cells per 100 basal keratinocytes, median values and quartiles)

\begin{tabular}{|c|c|c|c|c|c|c|c|}
\hline \multirow[b]{2}{*}{ Populations of cells } & \multirow[b]{2}{*}{ Healthy volunteers $(n=10)$} & \multicolumn{3}{|c|}{ Patients with vitiligo $(n=16)$} & \multirow[b]{2}{*}{$P_{1}$} & \multirow[b]{2}{*}{$P_{2}$} & \multirow[b]{2}{*}{$P_{3}$} \\
\hline & & Lesional skin & Marginal zone & $\begin{array}{l}\text { Area of perilesional normally } \\
\text { pigmented skin }\end{array}$ & & & \\
\hline Melan-A+ melanocytes & $10.9[9.6 ; 12.6]$ & $0[0 ; 1.0]$ & $1.0[0.2 ; 2.6]$ & $3.4[2.5 ; 5.2]$ & $<0.001$ & $<0.001$ & $<0.001$ \\
\hline $\mathrm{FM}+$ cells & $52.0[38.4 ; 100.2]$ & $1.0[0 ; 7.8]$ & $18.1[5.6 ; 55.9]$ & $54.6[40.0 ; 65.1]$ & $<0.001$ & 0.013 & 0.598 \\
\hline
\end{tabular}

Legend: Melan-A+ melanocytes: cells expressing Melan-A antigen (determination with A103 monoclonal antigens); FM+ cells: cells containing melanin granules (staining with the Fontana-Masson method); P1, P2, and P3: levels of statistical significance during comparison of parameters in healthy volunteers and patients, respectively, in lesional skin, the marginal zone, and the area of perilesional normally pigmented skin 
in melanocytes but also in adipocytes, Schwann cells, and myoepithelial cells $(24,26-30)$. The HMB-45 marker, which identifies gp10o (glycoprotein of premelanosomes), is considered more specific than the S-10o marker but it also does not show absolute specificity because it can be expressed in the cells of sweat glands and non-melanocytic tumors $(24,31,32)$. It also has less sensitivity than Melan-A (20, 23, 33).

The Melan-A marker also does not have absolute specificity for melanocytes and may show immunoreactivity for cells of nonmelanocytic origin: steroid-producing tumors, angiolipomes, and some others (18, 34-39). Moreover, Melan-A can be expressed by macrophages, but its immunohistochemical staining is weak and granulous, in contrast to the strong expression of melanocytic cells (40).

This study used the Melan-A marker (A103 clone) to identify melanocytes and evaluate their amount in various zones of nonsegmental vitiligo lesions. Our results indicate that the Melan-A marker is expressed both in the skin of healthy volunteers and in the skin of vitiligo patients. In vitiligo patients, Melan-A+ melanocytes and melanin granules (the sections were stained using the Fontana-Masson method) have been found in all the zones of vitiligo lesions.

The determined presence of Melan-A+ cells and melanin granules in depigmented skin of patients, including those with long-standing disease (up to 40 years), indicates that the residual melanocytes are preserved in vitiligo lesions. The data obtained appear to confirm the results of other authors. Tobin et al. also detected residual melanocytes in the epidermis of depigmented patients' skin even in cases of long-standing disease (up to 25 years) (8). Expression of tyrosinase was revealed in some of these cells by dopa reaction. Moreover, during ultrastructural analysis of depigmented skin, mature melanin granules were found in the basal and suprabasal layers of the epidermis in 10 of 12 patients examined. In the authors' opinion, these melanin granules were synthesized by residual melanocytes. Based on the results, the authors concluded that active (partly functioning) residual melanocytes are preserved in the depigmented skin of vitiligo patients.

Kim et al. investigated the lesional skin of 100 vitiligo patients and found the presence of melanin granules in $16 \%$ of the cases (staining with the Fontana-Masson method) and the expression of NKI-beteb+ melanocytes in $12 \%$ of the cases (9). De Francesco et al. found tyrosinase+ and HMB-45+ cells in lesional skin in three of 14 untreated vitiligo patients $(21.4 \%)$. These three patients displayed $>75 \%$ repigmentation after 9 months of narrow-band UVB phototherapy (41). Seleit et al. demonstrated the presence of HMB-45+ cells (indicating differentiated and active melanocytes) in $44 \%$ of cases in interfollicular epidermis and in $46.7 \%$ of cases in follicular epidermis in lesional vitiligo skin (10). In their latest work, they detected HMB-45+ melanocytes and TRP2+ (Tyrosinase Related Protein 2) melanocytes in $25 \%$ and $75 \%$ of vitiliginous white hair, respectively (42).

Our findings show that in patients with non-segmental vitiligo the pathological process may involve not only depigmented skin but normally pigmented, clinically intact skin as well. Our results are consistent with the results of studies by Le Poole et al., who found a more than two-fold decrease of NKI-beteb melanocytic marker expression (43), and by Tobin et al., who demonstrated the failure of maturation and transfer of melanosomes from melanocytes into keratinocytes of the basal and suprabasal layers of the epidermis in this area (8). Wańkowicz-Kalińska et al. revealed the association of microscopic signs of melanocyte destruction in clinically intact skin of patients with generalized vitiligo with skin infiltration with T-cells (44).

Recently, new data became available on defects in normalappearing skin of vitiligo lesions. Wagner et al. demonstrated altered adhesion between melanocytes and keratinocytes in the epidermis of vitiligo patients that, in turn, was linked to the absence of and discontinuous distributions of E-cadherin in clinically normal skin (45). Anbar et al. used electron microscopy to detect degenerative changes in perilesional melanocytes of non-segmental vitiligo patients in the form of vacuolization of the melanocytic cytoplasm, pyknosis of nuclei, and peripheral margination of chromatin (46). Ding et al. found that melanocytes from perilesional vitiligo skin contain few mitochondria (47). The structures of those mitochondria are irregular in various shapes and sizes, most of which are swollen with obscure cristae and vacuolization, especially in active vitiligo.

\section{Conclusion}

This study indicates the usefulness of the Melan-A marker for identifying melanocytes in non-segmental vitiligo patients' skin and for estimating their content in different zones of the vitiligo lesions. Expression of melanosomal protein Melan-A and melanin granules was found in all three zones of vitiligo lesions. For some patients, including those with long-standing disease (up to 40 years), Melan-A+ cells and melanin granules were detected in depigmented skin as an indication that the residual melanocytes are preserved in vitiligo lesions, and these results are consistent with the data of other authors. A greater than three-fold decrease in Melan-A+ melanocyte amount was revealed in perilesional normally pigmented skin of vitiligo patients, as compared to the skin of healthy volunteers. The possibility of clinically intact skin involvement in the pathological process should be taken into consideration if local treatment methods are prescribed.

\section{References}

1. Le Poole IC, Luiten RM. Autoimmune etiology of generalized vitiligo. Curr Dir Autoimmun. 2008;10:227-43.

2. Rezaei N, Gavalas NG, Weetman AP, Kemp EH. Autoimmunity as an aetiological factor in vitiligo. J Eur Acad Dermatol Venereol. 2007;21:865-76.

3. Oiso N, Suzuki T, Fukai K, Katayama I, Kawada A. Nonsegmental vitiligo and autoimmune mechanism. Dermatol Res Pract. 2011;2011:518090. doi: 10.1155/ 2011/518090.

4. Cui J, Shen L, Wang G. Role of hair follicles in the repigmentation of vitiligo. J Invest Dermatol. 1991;97:410-16.

5. Abdel-Naser MB, Krüger-Krasagakes S, Krasagakis K, Gollnick H, Abdel-Fattah A, Orfanos CE. Further evidence for involvement of both cell mediated and humoral immunity in generalized vitiligo. Pigment Cell Res. 1994;7:1-8.

6. Van den Wijngaard RM, Wankowicz-Kalinska A, Le Poole C, Tigges B, Westerhof $W$, Das P. Local immune response in skin of generalized vitiligo patients. Destruction of melanocytes is associated with the prominent presence of CLA+ T cells at the perilesional site. Lab Invest. 2000;80:1299-309.

7. Esmat SM, El-Tawdy AM, Hafez GA, Zeid OA, Abdel Halim DM, Saleh MA, et al. Acral lesions of vitiligo: why are they resistant to photochemotherapy? J Eur Acad Dermatol Venereol. 2012;26:1097-104.

8. Tobin DJ, Swanson NN, Pittelkow MR, Peters EM, Schallreuter KU. Melanocytes are not absent in lesional skin of long duration vitiligo. J Pathol. 2000;191:407-16.

9. Kim YC, Kim YJ, Kang HY, Sohn S, Lee ES. Histopathologic features in vitiligo. Am J Dermatopathol. 2008;30:112-6. 
10. Seleit I, Bakry OA, Abdou AG, Dawoud NM. Immunohistochemical study of melanocyte-melanocyte stem cell lineage in vitiligo; a clue to interfollicular melanocyte stem cell reservoir. Ultrastruct Pathol. 2014;38:186-98.

11. Pretti Aslanian F, Filgueira A, Cuzzi T, Vergier B. Histopathology. In: Picardo M, Taïeb A (eds.). Vitiligo.Springer-Verlag, Berlin; 2010; p. 25-32.

12. Busam KJ, Jungbluth AA. Melan-A, a new melanocytic differentiation marker. Adv Anat Pathol. 1999;6:12-8.

13. Hoashi T, Watabe H, Muller J, Yamaguchi Y, Vieira WD, Hearing VJ. MART-1 is re quired for the function of the melanosomal matrix protein PMEL17/GP100 and the maturation of melanosomes. J Biol Chem. 2005;280:14006-16.

14. Ordóñez NG. Value of melanocytic-associated immunohistochemical markers in the diagnosis of malignant melanoma: a review and update. Hum Pathol. 2014; 45:191-205.

15. Ogg GS, Dunbar PR, Romero P, Chen JL, Cerundolo V. High frequency of skin homing melanocyte-specific cytotoxic T lymphocytes in autoimmune vitiligo. Exp Med. 1998;188:1203-8.

16. Lang KS, Caroli CC, Muhm A, Wernet D, Moris A, Schittek B, et al. HLA-A2 restricted, melanocyte-specific CD8+ T lymphocytes detected in vitiligo patients are related to disease activity and are predominantly directed against MelanA/ MART1. J Invest Dermatol. 2001;116:891-7.

17. Teulings HE, Willemsen KJ, Glykofridis I, et al. The antibody response against MART-1 differs in patients with melanoma-associated leucoderma and vitiligo. Pigment Cell Melanoma Res. 2014;27:1086-96.

18. Prieto VG, Shea CR. Immunohistochemistry of melanocytic proliferations. Arch Pathol Lab Med. 2011;135:853-9.

19. De Vries TJ, Fourkour A, Wobbes T, Verkroost G, Ruiter DJ, van Muijen GN. Heterogeneous expression of immunotherapy candidate proteins gp100, MART-1 and tyrosinase in human melanoma cell lines and in human melanocytic lesions. Cancer Res. 1997;57:3223-9.

20. Fetsch PA, Marincola FM, Filie A, Hijazi YM, Kleiner DE, Abati A. Melanoma-associated antigen recognized by T cells (MART-1): the advent of a preferred immunocytochemical antibody for the diagnosis of metastatic malignant melanoma with fine-needle aspiration. Cancer. 1999;87:37-42.

21. Beaty MW, Fetsch P, Wilder AM, Marincola F, Abati A. Effusion cytology of malignant melanoma. A morphologic and immunocytochemical analysis including application of the MART-1 antibody. Cancer. 1997;81:57-63.

22. Orosz Z. Melan-A / Mart-1 expression in various melanocytic lesions and in nonmelanocytic soft tissue tumours. Histopathology. 1999;34:517-25.

23. Clarkson KS, Sturdgess IC, Molyneux AJ. The usefulness of tyrosinase in the immunohistochemical assessment of melanocytic lesions: a comparison of the novel T311 antibody (anti-tyrosinase) with S-100, HMB45 and A103 (anti-melanA). I Clin Pathol. 2001;54:196-200.

24. Sheffield MV, Yee H, Dorvault CC, Weilbaecher KN, Eltoum IA, Siegal GP. Comparison of five antibodies as markers in the diagnosis of melanoma in cytologic preparations. Am J Clin Pathol. 2002;118:930-6.

25. Fernandes BF, Odashiro AN, Saraiva VS, Logan P, Antecka E, Burnier MN Jr. Immunohistochemical expression of melan-A and tyrosinase in uveal melanoma. Carcinogenesis. 2007;6:1-6.

26. Fukai I, Masaoka A, Yamakawa Y, Niwa H, Eimoto T. Mediastinal malignant epithelioid schwannoma. Chest. 1995;108:574-5.

27. Seki K, Hasegawa T, Konegawa R, Hizawa K, Sano T. Primary liposarcoma of the stomach: a case report and a review of the literature. Japanese J Clin Oncol. 1998;28:284-8

28. Cheng L, Scheithauer BW, Leibovich BC, Ramnani DM, Cheville JC, Bostwick DG. Neurofibroma of the urinary bladder. Cancer. 1999;86:505-13.
29. Sundram U, Harvell JD, Rouse RV, Natkunam Y. Expression of the B-cell proliferation marker MUM1 by melanocytic lesions and comparison with $\mathrm{S}_{100}$, gp10o (HMB45) and MelanA. Mod Pathol. 2003;16:802-10.

30. Halawi A, Abbas 0 , Mahalingam M. S10o proteins and the skin: a review. J Eur Acad Dermatol Venereol. 2014;28:405-14.

31. Bonetti F, Colombari R, Manfrin E, Zamboni M, Martignoni G, Mombello A et al. Breast carcinoma positive for the melanoma marker (HMB45). HMB45 immunoreactivity in normal and neoplastic breast. Am J Clin Pathol. 1989;92:91-5.

32. Kanitakis J, Hermier C, Chouvet B, Thivolet J. Reactivity of HMB 45 monoclonal antibody with sweat gland tumours of the skin. Acta Derm Venereol. 1991;71:426-8.

33. Zubovits J, Buzney E, Yu L, Duncan LM. HMB-45, S-100, NK $1 / \mathrm{C}_{3}$ and MART-1 in metastatic melanoma. Hum Pathol. 2004;35:217-23.

34. Stewart CJR, Nandini CL, Richmond JA. Value of $\mathrm{A}_{103}$ (melan-A) immunostaining in the differential diagnosis of ovarian sex cord stromal tumours. J Clin Pathol. 2000;53:206-11.

35. Shin SJ, Hoda RS, Ying L, DeLellis RA. Diagnostic utility of the monoclonal an tibody $\mathrm{A}_{103}$ in fine-needle aspiration biopsies of the adrenal. Am J Clin Pathol. 2000;113:295-302.

36. Zhang PJ, Genega EM, Tomaszewski JE, Pasha TL, LiVolsi VA. The role of calretinin, inhibin, Melan-A, BCL-2 and c-kit in differentiating adrenal cortical and medullary tumors: an immunohistochemical study. Mod Pathol. 2003;16:591-7.

37. Smith-Zagone MJ, Prieto VG, Hayes RA, Timperman WW Jr, Diwan AH. HMB-45 (gp103) and MART-1 expression within giant cells in an atypical fibroxanthoma: a case report. J Cutan Pathol. 2004;31:284-6.

38. Parfitt JR, Bella AJ, Wehrli BM, Izawa JI. Primary PEComa of the bladder treated with primary excision and adjuvant interferon-alpha immunotherapy: a case report. BMC Urology. 2006;6:20.

39. Gleason BC, Nascimento AF, HMB-45 and Melan-A are useful in the differential diagnosis between granular cell tumor and malignant melanoma. Am J Dermatopathol. 2007;29:22-7.

40. Ferringer T. Update on immunohistochemistry in melanocytic lesions. Dermato Clin. 2012;30:567-79.

41. De Francesco V, Stinco G, Laspina S, Parlangeli ME, Mariuzzi L, Patrone P. Im munohistochemical study before and after narrow band (311 nm) UVB treatment in vitiligo. Eur J Dermatol. 2008;18:292-96.

42. Seleit I, Bakry OA, Abdou AG, Dawoud NM. Immunohistochemical evaluation of vitiliginous hair follicle melanocyte reservoir: is it retained? J Eur Acad Dermato Venereol. 2015;29:444-51.

43. Le Poole IC, van den Wijngaard R, Westerhof W, Das PK. Presence of T cells and macrophages in inflammatory vitiligo skin parallels melanocyte disappearance. Am J Pathol. 1996;148:1219-28.

44. Wańkowicz-Kalińska A, van den Wijngaard RM, Tigges BJ, Westerhof W, Ogg GS, Cerundolo V. Immunopolarization of $\mathrm{CD}_{4}+$ and CD8 + T cells to Type-1-like is associated with melanocyte loss in human vitiligo. Lab Invest. 2003;83:683-95.

45. Wagner RY, Luciani F, Cario-André M, et al. Altered E-Cadherin levels and distribution in melanocytes precede clinical manifestations of vitiligo. J Invest Dermatol. 2015;135:1810-9.

46. Anbar TS, El-Sawy AE, Attia SK, et al. Effect of PUVA therapy on melanocytes and keratinocytes in non-segmental vitiligo: histopathological, immuno-histochemical and ultrastructural study. Photodermatol Photoimmunol Photomed. 2012;28:17-25.

47. Ding GZ, Zhao WE, Li X, Gong QL, Lu Y. A comparative study of mitochondrial ultrastructure in melanocytes from perilesional vitiligo skin and perilesional halo nevi skin. Arch Dermatol Res. 2015;307:281-9 